\title{
A Method for Estimating the Environmental Impact of a Wind Farm by Fuzzy Logic Techniques
}

\author{
G. Delvecchio \\ Technical Area \\ University of Bari \\ Piazza Umberto I n.1, 70100 Bari (Italy) \\ phone: +39 080 5714648, fax: +39080 5714655, e-mail: g.delvecchio@area-tecnica.uniba.it
}

\begin{abstract}
A very interesting question in studying the problems connected with wind farms is undoubtedly their impact on the landscape in which they are installed. Among the different methods for evaluating this impact, that defined by Électricité de France (EDF) has been taken into examination in this paper.

This study aims at improving the EDF method by means of Fuzzy Logic techniques. This results in a new method that implies a clear simplification in the application of the EDF method.
\end{abstract}

\section{Key words}

Fuzzy logic, environmental impact, wind farm.

\section{Introduction}

The concepts of "landscape" based on purely and simply aesthetic criteria are nowadays obsolete as there is a general inclination to define the landscape according to the concepts of environment and territory [1] - [5]. The landscape gives birth to many natural and artificial "marks", and supplies the people who live and work there with a historical, social, technological importance, etc. (think, for example, to the skyscrapers of a large metropolitan area) [6].

In this context a wind farm can be certainly considered as a "mark" representing not only a technological improvement, but also a historical, social awareness of the environmental, energy problems. However this new way of interpreting the "landscape" must not be detrimental to the role of the pre-existent elements of the same landscape [6].

The estimation of the environmental impact depends exactly on the consideration of what is likely to happen to the pre-existent elements when a wind farm is installed.

Among the different ways of dealing with this problem, the approach defined by Électricité de France $(E D F)$ has nowadays proven to be successful [1], [4].

In this paper the Author suggests that the abovementioned method applied to wind farms can be improved by means of Fuzzy techniques.

In particular he will:

1) give a brief account of the EDF method;

2) show how the application of this method is rather difficult;

3) illustrate the new fuzzy approach suggested;
4) apply the EDF method to an Italian wind farm;

5) apply the fuzzy method suggested to this wind farm;

6) compare the results.

\section{The method defined by EDF}

As is well-known, this method was initially conceived for evaluating the visual impact of power lines, but it has turned out to be right for wind farms, too [6]. The EDF method is based on the analysis of the landscape where the wind farm is installed, considering that it could somehow have an environmental effect on the landscape. The landscape is therefore analyzed under two frames of reference, that is "visual quality" and "picturesque quality".

The visual quality of the landscape is defined as the ability of the landscape to limit the visual impact of a building by making it not very visible. The components of the visual quality are: width of the view (or scale of vision), scale of the visual elements of the landscape (or inner scale), their readability and complexity.

TABLE I - Categories of the components of the visual quality and their corresponding index [1], [4].

\begin{tabular}{|l|c|}
\hline \multicolumn{1}{|c|}{ Category } & Index \\
\hline Great scale of vision & 1 \\
Great inner scale & \\
Great readability & \\
Small complexity & 1 \\
\hline Small scale of vision & \\
Small inner scale & \\
Small readability & \\
Great complexity & \\
\hline Great scale of vision & \\
Medium inner scale & \\
Great readability & \\
Medium complexity & \\
\hline Medium scale of vision & 2 \\
Small inner scale & \\
Medium readability & \\
Great complexity & \\
\hline Medium scale of vision & \\
Medium inner scale & \\
Medium readability & \\
Medium complexity & \\
\hline
\end{tabular}

On the contrary, the picturesque quality of the landscape classifies the ecological, aesthetic and historical peculiarities of the landscape with respect to their sensitivity to man-made interventions. The picturesque quality is char- 
acterized by the degree to which its components are banal, artificial, variable, innovatory and different.

\section{A. Visual quality}

In the EDF method there are five possible categories for the components of the visual quality, and each category has a corresponding index, according to TABLE I.

\section{B. Picturesque quality}

Each component of the picturesque quality is subdivided into three categories, and each of them has a corresponding score $(-1,0,1)$ according to TABLE II.

TABLE II - Components of the picturesque quality, their subdivision into categories and their corresponding score [1], [4].

\begin{tabular}{|l|c|c|c|}
\hline Degree & $\mathbf{1}$ & $\mathbf{0}$ & $\mathbf{- 1}$ \\
\hline Degree of banality & Uncommon & Mixed & Common \\
\hline Degree of artificiality & Natural & Mixed & Artificial \\
\hline Degree of variation & Unchangeable & Mixed & Changeable \\
\hline Degree of innovation & Old & Mixed & New \\
\hline Degree of diversity & $\begin{array}{c}\text { Meeting with } \\
\text { opposition }\end{array}$ & Mixed & $\begin{array}{c}\text { Monoto- } \\
\text { nous }\end{array}$ \\
\hline
\end{tabular}

The sum of these scores leads to the definition of three categories of sensitivity, each having an index according to TABLE III.

TABLE III - Categories of the picturesque quality and their corresponding index.

\begin{tabular}{|l|c|c|}
\hline \multicolumn{1}{|c|}{ Scores } & Category & Index \\
\hline From -5 to -3 & Positive & 1 \\
\hline From -2 to +2 & Neutral & 2 \\
\hline From +3 to +5 & Negative & 3 \\
\hline
\end{tabular}

\section{Environmental impact}

According to the previous tables, it is possible to get two indices associated with the two above-mentioned different qualities, respectively.

The sum of the indices relating to the two aforesaid different qualities gives the three degrees of sensitivity (TABLE IV) taken by the landscape when a wind farm is installed. In other words, each degree of sensitivity is an indicator of the likely environmental impact of the wind farm on the landscape.

TABLE IV - Degree of sensitivity of the landscape when a wind farm is installed related to the sum of the indices of visual and picturesque qualities.

\begin{tabular}{|c|c|}
\hline $\begin{array}{c}\text { Sum of the two } \\
\text { indices }\end{array}$ & $\begin{array}{c}\text { Degree of sensitivity } \\
\text { of the landscape }\end{array}$ \\
\hline 2 or 3 & Low sensitive \\
\hline 4 & Medium-sensitive \\
\hline 5 or 6 & Very sensitive \\
\hline
\end{tabular}

\section{The Fuzzy method suggested}

In the EDF method it is difficult, according to the tables available, to determine with absolute precision the indices relating to the picturesque and visual qualities, as well as it is difficult to get the exact degree of sensitivity of the landscape. For, the degree of sensitivity of the landscape can not be strictly classified only in one of the three categories of TABLE IV, but it is reasonable to suppose that these three categories do not have clear outlines, but they are veiled, blurred, fuzzy. The fuzzy logic [7] can therefore simplify the approach to the method, making its application much easier. So let's give a brief account of this method.

In the method here suggested (which is an improvement of the paper [8]), the Author starts establishing a score between 0 and 1 for each component of the visual and picturesque quality: 0 represents the minimum value of the component and 1 the maximum value. To simplify, this score is indicated by the variables $v_{i}$ and $p_{i}$ in TABLE $\mathrm{V}$ and TABLE VI, for the visual and picturesque qualities, respectively.

TABLE V - Components of the visual quality and their score.

\begin{tabular}{|l|c|}
\hline \multicolumn{1}{|c|}{ Components of the visual quality } & score \\
\hline Scale of vision & $v_{1}$ \\
\hline Inner scale & $v_{2}$ \\
\hline Readability & $v_{3}$ \\
\hline Complexity & $v_{4}$ \\
\hline
\end{tabular}

TABLE VI - Components of the picturesque quality and their score.

\begin{tabular}{|l|c|}
\hline \multicolumn{1}{|c|}{ Components of the picturesque quality } & score \\
\hline Degree of banality & $p_{1}$ \\
\hline Degree of artificiality & $p_{2}$ \\
\hline Degree of variation & $p_{3}$ \\
\hline Degree of innovation & $p_{4}$ \\
\hline Degree of diversity & $p_{5}$ \\
\hline
\end{tabular}

For example, an average "Scale of vision" can be associated with $v_{1}=0.5$, while a middle/high "Degree of innovation" can be associated with $p_{4}=0.75$.

The fuzzy method consists of the following steps:

1) the memberships to be applied to both the visual and picturesque quality are determined (as shown in Fig. 1);

2) by the memberships used for the visual quality and the scores $v_{i}$ of TABLE $\mathrm{V}$, the $V Q$ index associated with the visual quality is calculated (as shown in Fig. 2);

3) by the memberships used for the picturesque quality and the scores $p_{i}$ of TABLE VI, the $P Q$ index associated with the picturesque quality is calculated (as shown in Fig. 3);

4) $V Q$ and $P Q$ allow to calculate (by means of (1)) the output of the fuzzy approach, that is the $Q$ index associated with the environmental impact; 
5) $Q$ allows to calculate the membership degree of the sensitivity of the landscape to the "Small", "Medium" and "Great" categories (Fig. 4).

Let's go into the above steps.

\section{A. Memberships used for visual and picturesque quality}

As is well-known, in the theory of fuzzy sets the membership of an element of a fuzzy set is given by a membership function whose values are between 0 and 1 .

In the case under examination we have used memberships like those of Fig. 1 associated with the "small" $(S)$, "medium" $(M)$ and "great" $(G)$ variables, both for the visual and picturesque qualities. Similar memberships have also been used for the indices 1, 2 and 3 (see TABLE I and TABLE III).

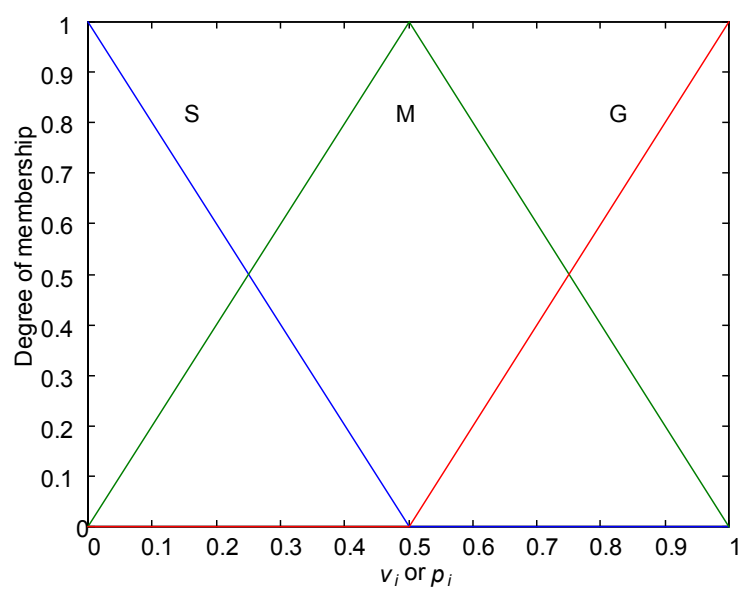

Fig. 1. Representation of the memberships associated with the $S, M, G$ variables, respectively, of the components of the visual and picturesque quality.

\section{B. Visual quality}

In accordance with TABLE I, each category given in this table follows the fuzzy rules in the following way:

1) If the "scale of vision" is GREAT \& the "inner scale" is GREAT \& the "readability" is GREAT \& the "complexity" is SMALL, then the index is 1 . This rule is shown in the first line of the diagrams in Fig. 2. To each of the 4 diagrams of this line in abscissa correspond the scores $v_{i}$ of TABLE X, these scores being relating to the example of section 5 .

2) If the "scale of vision" is SMALL \& the "inner scale" is SMALL \& the "readability" is SMALL \& the "complexity" is GREAT, then the index is 1 (see the second line of the diagrams in Fig. 2).

3) If the "scale of vision" is GREAT \& the "inner scale" is MEDIUM \& the "readability" is GREAT $\&$ the "complexity" is MEDIUM, then the index is 2 (see the third line of the diagrams in Fig. 2).

4) If the "scale of vision" is MEDIUM \& the "inner scale" is SMALL \& the "readability" is MEDIUM $\&$ the "complexity" is GREAT, then the index is 2 (see the fourth line of the diagrams in Fig. 2).
5) If the "scale of vision" is MEDIUM \& the "inner scale" is MEDIUM \& the "readability" is MEDIUM $\&$ the "complexity" is MEDIUM, then the index is 3 (see the fifth line of the diagrams in Fig. 2).

Consequently the index $V Q$ to be found, associated with the visual quality, is determined by the "Correlation Minimum Encoding" rule [7] (see the last column in Fig. 2). It should be noted that $V Q$ varies from 1 and 3 .

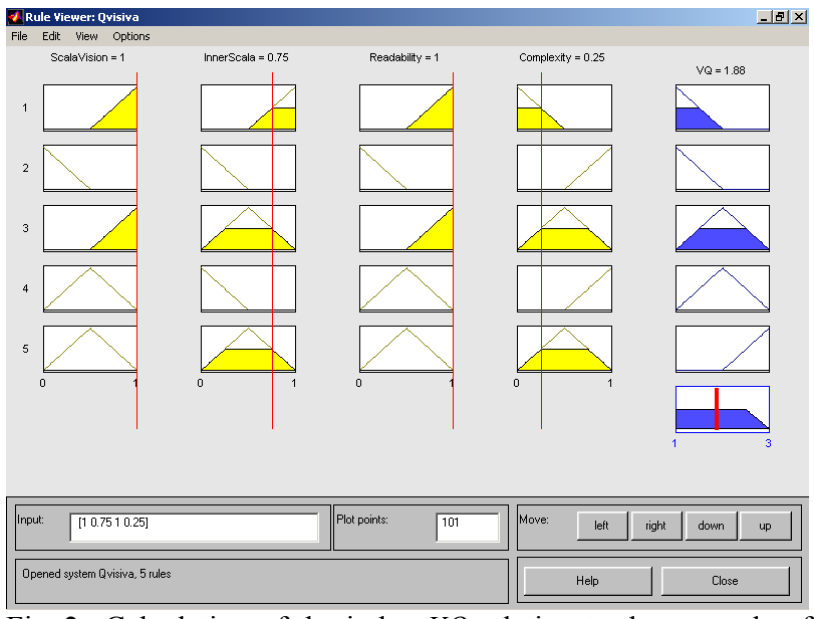

Fig. 2. Calculation of the index $V Q$ relating to the example of section 5 .

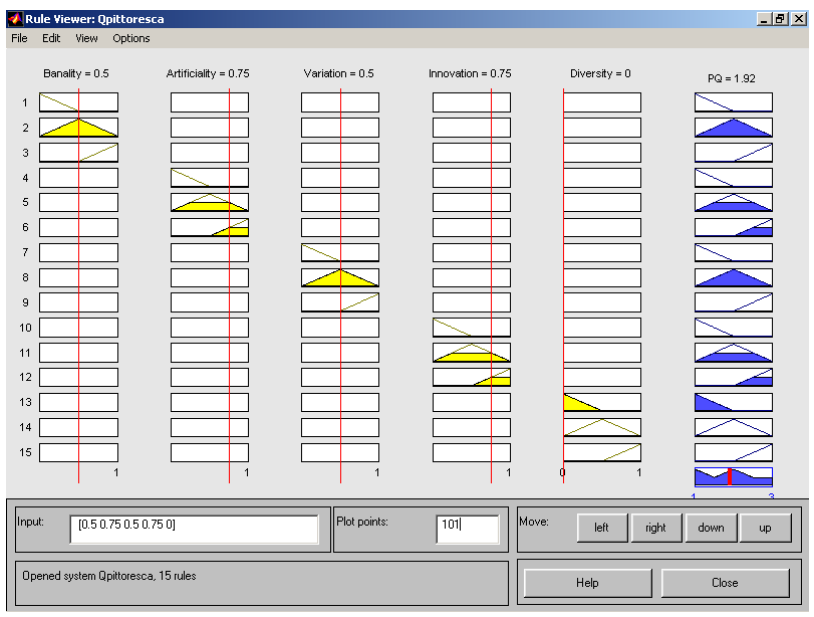

Fig. 3. Calculation of the index $P Q$ relating to the example of section 5 .

\section{Picturesque quality}

From TABLE II and TABLE III the Author has inferred 3 rules for each of the 5 components of the picturesque quality.

Let's consider, for example, the $1^{\text {st }}$ component of the picturesque quality: the "Degree of banality". The fuzzy rules to be associated with it are the following (cf. TABLE II and TABLE III):

1) If the "Degree of banality" is SMALL, then the index is 1 . This rule is shown in the first line of the diagrams in Fig. 3. To each of the 5 diagrams of this line in abscissa correspond the scores $p_{i}$ of 
TABLE XI, these scores being relating to the example of section 5 .

2) If the "Degree of banality" is MEDIUM, then the index is 2 (see the second line of the diagram in Fig. 3).

3) If the "Degree of banality" is GREAT, then the index is 3 (see the third line of the diagram in Fig. 3).

The same is true of the other 4 components of the picturesque quality. In total we have 15 rules (see Fig. 3 ).

Consequently, as already seen in the previous case, the index $P Q$ to be found, associated with the picturesque quality, is determined by the "Correlation Minimum Encoding" rule [7] (see the last column in Fig. 3). It should be noted that $P Q$ varies between 1 and 3 .

\section{Environmental impact}

The sum of the indices relating to the two different qualities gives the index $Q$, corresponding to the environmental impact, according to the following equation.

$$
Q=V Q+P Q
$$

It is worth noting that $Q$ varies between 2 and 6 .

\section{E. The membership degree of the kind of sensitivity of the landscape}

The value of $Q$ calculated by (1) allows the Author to determine, by means of a suitable membership, the degree of membership of the landscape to the three categories shown in TABLE VII. The membership function:

$$
\mu(x)=\frac{1}{1+\left(\frac{x-m}{\alpha}\right)^{2}}
$$

has been used [9], where:

$m$ : is the mean of the membership,

$\alpha$. is the width of the membership.

From TABLE IV the Author has inferred the memberships of the categories of sensitivity of the landscape according to the parameters given in TABLE VII. These memberships have been represented in Fig. 4.

TABLE VII - Categories of sensitivity of the landscape, and the relative parameters of the memberships.

\begin{tabular}{|l|c|c|c|}
\hline Degree of sensitivity of the landscape & Membership & $\mathbf{m}$ & $\boldsymbol{\alpha}$ \\
\hline Low sensitive (Small - S) & $\mu_{S}(x)$ & 2 & 1 \\
\hline Medium sensitive (Medium - M) & $\mu_{M}(x)$ & 4 & 1 \\
\hline Very sensitive (Great - G) & $\mu_{G}(x)$ & 6 & 1 \\
\hline
\end{tabular}

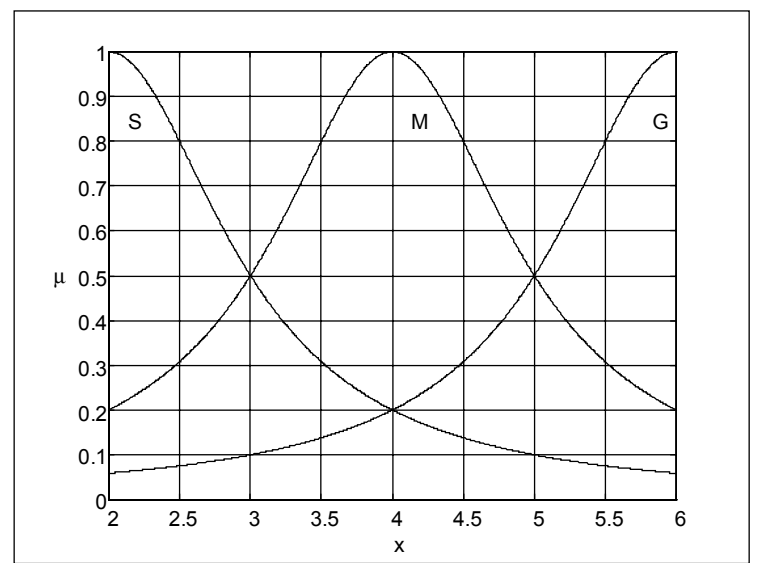

Fig. 4. Representation of the memberships relating to the categories of sensitivity of the landscape, according to TABLE VII.

The value of $Q$ calculated by (1) allows the Author to determine the degree of membership of the landscape to the three categories given in TABLE VII. The final output of the fuzzy method suggested is the following vector.

$$
\left[\mu_{S}(Q), \quad \mu_{M}(Q), \quad \mu_{G}(Q)\right]
$$

\section{The EDF method applied to an Italian wind farm}

The wind farm installed in Bisaccia (Italy) has been taken into examination in this paper and its environmental impact has been estimated by both the EDF method and the fuzzy method suggested by the Author.

The results coming from the application of the EDF method to the wind farm of Bisaccia found in [6] are here given.

As far as the visual quality (whose components are given in TABLE VIII) is concerned, a medium/great capacity of visual integration characterized by an index 1.5 (see TABLE I) has been found in [6] by the EDF method.

TABLE VIII -Components of the visual quality in the case of the wind farm of Bisaccia [6].

\begin{tabular}{|l|c|}
\hline \multicolumn{1}{|c|}{ Components } & Integration capacity of the landscape \\
\hline Scale of vision & Great \\
\hline Inner scale & Medium/Great \\
\hline Readability & Great \\
\hline Complexity & Small/Medium \\
\hline
\end{tabular}

As far as the picturesque quality (whose components are given in TABLE IX) is concerned, a score equal to zero (medium picturesque quality) with an index equal to 2 corresponding to a neutral landscape (see TABLE II and TABLE III) has been found in [6] by the EDF method. 
TABLE IX - Components of the picturesque quality and their relative score in the case of the wind farm of Bisaccia [6].

\begin{tabular}{|l|c|c|}
\hline Components & Value & Score \\
\hline Degree of banality & Mixed & 0 \\
\hline Degree of artificiality & Natural/Mixed & 0.5 \\
\hline Degree of variation & Mixed & 0 \\
\hline Degree of innovation & Old/Mixed & 0.5 \\
\hline Degree of diversity & Monotonous & -1 \\
\hline
\end{tabular}

The sum of the above two indices of quality gives a value equal to 3.5 which corresponds to a medium-low sensitivity of the landscape (see TABLE IV).

According to the Author, the application of the EDF method to the wind farms turns out to be hard. Moreover, it is not clear if the case under examination belongs to a category of visual quality of TABLE I, especially, for example, if the "Inner scale" is very close to «medium»" than to «great».

\section{The fuzzy method applied to an Italian wind farm}

Let's assign to each component of the visual and picturesque quality a score between 0 and 1 , given in TABLE $X$ and TABLE XI, respectively.

TABLE X - Components of the visual quality and their score in the case of the wind farm of Bisaccia.

\begin{tabular}{|l|c|l|}
\hline Degree & Value TABLE VIII & \multicolumn{1}{c|}{ Score } \\
\hline Scale of vision & Great & $v_{1}=1$ \\
\hline Inner scale & Medium/Great & $v_{2}=0.75$ \\
\hline Readability & Great & $v_{3}=1$ \\
\hline Complexity & Small/Medium & $v_{4}=0.25$ \\
\hline
\end{tabular}

TABLE XI - Components of the picturesque quality and their score in the case of the wind farm of Bisaccia.

\begin{tabular}{|l|c|l|}
\hline Components & Value TABLE IX & \multicolumn{1}{c|}{ Score } \\
\hline Degree of banality & Mixed & $p_{1}=0.5$ \\
\hline Degree of artificiality & Natural/Mixed & $p_{2}=0.75$ \\
\hline Degree of variation & Mixed & $p_{3}=0.5$ \\
\hline Degree of innovation & Old/Mixed & $p_{4}=0.75$ \\
\hline Degree of diversity & Monotonous & $p_{5}=0$ \\
\hline
\end{tabular}

The application of the method given in section 3 leads to $V Q=1.8759$ (see Fig. 2), $P Q=1.9214$ (see Fig. 3), and consequently $Q=3.7973$ (see (1)).

The value of $Q$ calculated by (1) allows the Author to determine the degree of membership of the landscape to the three categories of sensitivity, that is "small", "medium" and "great" (see (3)), given by the following vector

$$
[0,236 \quad 0,961 \quad 0,171]
$$

that is to say, a medium-low sensitivity of the landscape, as found by the EDF method.

\section{Comparison of the results}

As can be seen from the comparison of the two methods, the value of the visual quality index is $V Q=1.8759$, while the value of the picturesque quality index is $P Q=1.9214$, which differ from 1.5 and 2, respectively, these being the values calculated by the EDF method: there's nothing surprising about it, since the Fuzzy method varies in the continuum and not in the discrete quantity as the EDF method.

Moreover, thanks to the fuzzy approach we can better understand that the sensitivity of the landscape tends more to the "medium" value than to the "low" value, since the fuzzy method, unlike the EDF method, takes all information given in TABLE VIII and TABLE IX into account (for example, the "slight complexity" of the visual quality, see TABLE VIII, which influences the index of TABLE III, and so $V Q$ ).

\section{Conclusions}

Starting from the EDF method used for estimating the environmental impact of the wind farms, the Author suggests in this paper a new method based on the Fuzzy logic.

Both methods have been applied to an Italian wind farm: the comparison shows not only that the application of the method suggested is a little easier than the EDF method but also that the method takes into account all the information lost with the EDF method.

\section{References}

[1] G. Gisotti, S. Bruschi, Valutare l'ambiente, La Nuova Italia Scientifica, Roma (1990).

[2] Direttiva CEE 27/06/1985 No. 85/337, La valutazione dell'impatto ambientale di determinati progetti pubblici e privati.

[3] Direttiva CEE 03/03/1997 No. 97/11, Modificazioni alla direttiva 85/337/CEE concernente la valutazione dell'impatto ambientale di determinati progetti pubblici e privati.

[4] Électricité de France, Ligne de transport d'énergie Chapernay - St. Maurice l'Exil, 2 Circuits à $400 \mathrm{kV}$, étude d'impact (1980).

[5] M. Zambrini, Indici e Scale di qualità paesaggistica, Valutazione di Impatto Ambientale, FAST, Milano (1993), pp. 415-461.

[6] A. Ambrosini, I. Sebastianelli, Inserimento nell'ambiente di una centrale eolica: Bisaccia, in Proc. Energia dal vento: aspetti tecnologici ed economici, Bari, pp. 65-78, 1994.

[7] B. Kosko, Neural networks and fuzzy systems: a dynamical systems approach to machine intelligence, Englewood Cliffs, NJ, Prentice Hall (1992).

[8] G. Delvecchio, Un approccio Fuzzy per la valutazione dell'impatto sul paesaggio di una centrale eolica, DEE, Polytechnic of Bari, report No. 12/99/S (1999).

[9] Y. Y. Hsu, H. C. Kuo, Distribution system load estimation and service restoration using a fuzzy set approach, IEEE Transaction on Power Delivery, vol. 8 n. 4, pp. 1950-1957, 1993. 